\title{
Reviewer's comment concerning "Direct repair of displaced anterior arch fracture of the atlas under microendoscopy: experience with seven patients" (DOI 10.1007/s00586-011-1965-5 R1 by Jian Wang)
}

\author{
Charles A. Sansur
}

Received: 1 September 2011 / Published online: 16 September 2011

(C) Springer-Verlag 2011

I would like to commend Dr. Wang Jian for his interesting work on the repair of displaced anterior arch fractures of the atlas using microendoscopy. While Dr. Jian's technique did not result in significant complications in this series of seven patients, we must remind our readers that surgical intervention is seldom required in patients with fractures of the $\mathrm{C} 1$ arch. Hence, surgical repair of the anterior arch fracture is controversial. With further experience in the treatment of these fractures, and with a potential randomized clinical trial one may potentially advocate this surgical technique if the surgical group will demonstrate superior results.
C. A. Sansur $(\square)$

Department of Neurosurgery, University of Maryland School of Medicine, 22 S. Greene St., Baltimore, MD 21201, USA

e-mail: csansur@smail.umaryland.edu 